\section{Simplified Evaluation of APSK Error Performance}

\section{O. Afelumo, A.B. Awoseyila and B.G. Evans}

An analytical evaluation of the performance of amplitude phase shift keying constellations in additive white Gaussian noise is performed and an expression to approximate the error probability is presented. The expression accounts for a number of symbols, equal to the number of concentric rings in the constellation. It is shown to significantly reduce the computational complexity associated with previously known error performance expressions, while closely predicting the error rates in the relevant signal-to-noise ratio range.

Introduction: Amplitude phase shift keying (APSK) is a higher-order modulation scheme designed for efficient transmission over satellite channels due to its intrinsic robustness against non-linear amplifier distortions, as well as spectral efficiency. The second generation standard for digital video broadcasting via satellite (DVB-S2) adopts the APSK scheme in conjunction with other features [1].

A union bound for the error performance of M-ary modulations in additive white Gaussian noise (AWGN) has been presented in [2]. This bound acts an upper limit for the symbol error rate of APSK modulations. In [3], the error performances of 16- and 32-APSK schemes were analyzed and tight error rate expressions were proposed. The expressions were derived based on symmetry and by including only the necessary error terms associated with the pair-wise probabilities of the symbols in the first quadrant of the constellations.

In this paper, we further simplify the computational approach used in [3] and obtain good error rate approximations for the symbol error rate (SER) and bit error rate (BER) performance of APSK schemes in AWGN, as applied to the 64-APSK constellation.

APSK error performance: The union bound for the symbol error rate of $M$-ary modulation schemes, as stated in [2], is given in general terms as:

$$
P(E)=\frac{1}{M} \sum_{i=1}^{M} P\left(E \mid s_{i}\right) \leq \frac{1}{M} \sum_{i=1}^{M} \sum_{j=1, j \neq i}^{M} P\left(s_{i} \rightarrow s_{j}\right)
$$

where $E$ denotes the error event, $P\left(s_{i} \rightarrow s_{j}\right)$ denotes the pair-wise probability that a transmitted symbol $s_{i}$ is erroneously detected as $s_{j}$ and $M$ denotes the order of the constellation. The pair-wise probability can be computed using the $Q$-function, expressed in terms of the Euclidean distance $d_{i j}$ and the single-sided noise spectral density $N_{o}$, as:

$$
P\left(s_{i} \rightarrow s_{j}\right)=Q\left(d_{i j} / \sqrt{2 N_{o}}\right)
$$

The union bound in (1) would however involve computing the error events for all the symbols in the constellation which would be cumbersome as the constellation order increases. Furthermore, the computation would include a considerable amount of overlap for the detection error regions, thereby increasing the gap between the error bound and the actual symbol error rate [3].

The approach used in [3] exploits the symmetry in the APSK constellations to simplify (1) while taking into consideration only the necessary error terms associated with the symbols used for computing the pair-wise probabilities, in order to avoid redundant error regions as is the case with the union bound in (1). However this approach becomes increasingly complicated as the constellation order increases.

Proposed method: In order to further simplify the evaluation of APSK error performance, we analyze the constellation using the concept of Voronoi diagram [4]. An optimum demodulator (assuming maximum likelihood (ML) detection) works with Voronoi cells as decision regions as shown in fig. 1 for the 64-APSK constellation. A close examination of this diagram also shows that the decision boundaries between different constellation rings can be approximated by concentric circles (dashed circles in fig. 1). Since all symbols in an APSK constellation ring are uniformly spaced, they have the same probability of being erroneously detected to symbols on the same ring, and they also have the same distance relationship to a circular decision boundary. Therefore, using an approximation of decision boundaries to concentric circles, it can be assumed that all symbols on a constellation ring have similar probability of being erroneously detected to the symbols on other rings. Consequently, the pair-wise error probability of a single symbol per ring is sufficient to approximate the error performance of APSK schemes.

Based on this, we can simplify the computational approach proposed in [3], and obtain an SER expression which involves only a limited number of symbols, equal to the number of rings in the constellation such that the final error probability $P(E)$ is given as:

$$
P(E)_{A P S K}=\frac{1}{m} \sum_{l=1}^{N} P\left(E \mid s_{k}\right) * n_{l} \quad l=1 \ldots N
$$

where, $N$ is the total number of rings in the constellation, $n_{l}$ denotes the number of symbols on the $l^{\text {th }}$ ring in one quadrant of the constellation, $k$ denotes the symbol subscript, $m=\sum_{l}^{N} n_{l}$ and $P\left(E \mid s_{k}\right)$ denotes the pairwise probability for the $k^{\text {th }}$ symbol.

SER and BER of 64-APSK: The 64-APSK nomenclature used in this paper is as defined in [5]. It is characterized by four constellation rings having 4, 12, 20 and 28 uniformly-spaced PSK points with radius $R_{l}$, $R_{2}, R_{3}$ and $R_{4}$ respectively. The radius ratios are denoted as:

$$
\gamma_{1}=R_{2} / R_{1}, \gamma_{2}=R_{3} / R_{1}, \gamma_{3}=R_{4} / R_{1}
$$

Radius ratios for the 64-APSK scheme, based on the maximum mutual information optimization criterion, are given in [6]. The average symbol energy of the constellation is given as:

$$
E_{S}=\left(1+3 \gamma_{1}{ }^{2}+5{\gamma_{2}}^{2}+7{\gamma_{3}}^{2}\right) R_{1}{ }^{2} / 16=\alpha{R_{1}}^{2}
$$

where $\alpha=\left(1+3 \gamma_{1}{ }^{2}+5 \gamma_{2}{ }^{2}+7 \gamma_{3}{ }^{2}\right) / 16$.

Applying the proposed method to 64-APSK as shown in fig. 1, wherein the subscript $k$ is chosen such that the set of symbols included in (3) conform to the same phase in one quadrant of the constellation, the symbol error probability can be written as:

$$
\begin{aligned}
P(E)=\frac{1}{16} \sum_{l=1}^{4} P\left(E \mid s_{k}\right) * n_{l}= & \frac{1}{16}\left[P\left(E \mid s_{1}\right)+3 P\left(E \mid s_{3}\right)\right. \\
& \left.+5 P\left(E \mid s_{7}\right)+7 P\left(E \mid s_{13}\right)\right]
\end{aligned}
$$

The necessary error events for the chosen symbols in (6) are given in terms of pair-wise probabilities as:

$$
\begin{aligned}
P\left(E \mid s_{1}\right)= & P\left(s_{1} \rightarrow s_{2}\right)+P\left(s_{1} \rightarrow s_{3}\right)+P\left(s_{1} \rightarrow s_{4}\right)+P\left(s_{1} \rightarrow s_{15}\right) \\
& +P\left(s_{1} \rightarrow s_{16}\right) \\
P\left(E \mid s_{3}\right)= & P\left(s_{3} \rightarrow s_{1}\right)+P\left(s_{3} \rightarrow s_{2}\right)+P\left(s_{3} \rightarrow s_{4}\right)+P\left(s_{3} \rightarrow s_{7}\right) \\
P\left(E \mid s_{7}\right)= & P\left(s_{7} \rightarrow s_{3}\right)+P\left(s_{7} \rightarrow s_{6}\right)+P\left(s_{7} \rightarrow s_{8}\right)+P\left(s_{7} \rightarrow s_{13}\right) \\
P\left(E \mid s_{13}\right)= & P\left(s_{13} \rightarrow s_{7}\right)+P\left(s_{13} \rightarrow s_{12}\right)+P\left(s_{13} \rightarrow s_{14}\right)
\end{aligned}
$$

In order to use the Q-function, the relevant Euclidean distances obtained from the geometry of the constellation can be shown to satisfy:

$$
\begin{aligned}
& d_{1,2}=\sqrt{R_{1}^{2}+R_{2}^{2}-2 R_{1} R_{2} \cos \left(\frac{\pi}{6}\right)}, d_{3,7}=R_{3}-R_{2}, d_{1,3}=R_{2}-R_{1} \\
& d_{7,13}=R_{4}-R_{3}, d_{1,15}=\sqrt{2} R_{1}, d_{3,2}=\left(2 \sin \frac{\pi}{12}\right) R_{2} \\
& d_{7,6}=\left(2 \sin \frac{\pi}{20}\right) R_{3}, d_{13,12}=\left(2 \sin \frac{\pi}{28}\right) R_{4}
\end{aligned}
$$

Substituting (2), (5), and (7-11) into (6) and making use of the distance relations $\quad d_{1,2}=d_{1,4}, \quad d_{1,15}=d_{1,16}, \quad d_{3,2}=d_{3,4}, \quad d_{7,6}=d_{7,8} \quad$ and $d_{13,12}=d_{13,14}$, we obtain an approximate SER expression for 64-APSK, given as: 


$$
\begin{aligned}
S E R_{64 A P S K} \leq & \frac{1}{8} Q\left(\sqrt{\frac{\left(1+\gamma_{1}^{2}-2 \gamma_{1} \cos \frac{\pi}{6}\right)}{2 \alpha} \frac{E_{S}}{N_{o}}}\right)+\frac{1}{4} Q\left(\sqrt{\frac{\left(\gamma_{1}-1\right)^{2}}{2 \alpha} \frac{E_{S}}{N_{o}}}\right)+\frac{1}{8} Q\left(\sqrt{\frac{1}{\alpha} \frac{E_{S}}{N_{o}}}\right) \\
& +\frac{3}{8} Q\left(\sqrt{\frac{2 \gamma_{1}^{2} \sin ^{2} \frac{\pi}{12}}{\alpha} \frac{E_{S}}{N_{o}}}\right)+\frac{1}{2} Q\left(\sqrt{\frac{\left(\gamma_{2}-\gamma_{1}\right)^{2}}{2 \alpha} \frac{E_{S}}{N_{o}}}\right)+\frac{5}{8} Q\left(\sqrt{\frac{2 \gamma_{2}^{2} \sin ^{2} \frac{\pi}{20}}{\alpha} \frac{E_{S}}{N_{o}}}\right) \\
& +\frac{3}{4} Q\left(\sqrt{\frac{\left(\gamma_{3}-\gamma_{2}\right)^{2}}{2 \alpha} \frac{E_{S}}{N_{o}}}\right)+\frac{7}{8} Q\left(\sqrt{\frac{2 \gamma_{3}^{2} \sin ^{2} \frac{\pi}{28}}{\alpha} \frac{E_{S}}{N_{O}}}\right)
\end{aligned}
$$

Extending the analysis in [3] to 64-APSK, the symbol error probability would be given as:

$$
\begin{aligned}
P(E)= & \frac{1}{16}\left(P\left(E \mid s_{1}\right)+2 P\left(E \mid s_{2}\right)+P\left(E \mid s_{3}\right)+2 P\left(E \mid s_{5}\right)+2 P\left(E \mid s_{6}\right)\right. \\
& +P\left(E \mid s_{7}\right)+2 P\left(E \mid s_{10}\right)+2 P\left(E \mid s_{11}\right)+2 P\left(E \mid s_{12}\right)+P\left(E \mid s_{13}\right)
\end{aligned}
$$

This requires evaluating (by inspection) the conditional error probabilities of 10 symbols (as compared to 4 in the proposed method), resulting in the computation of 14 Euclidean distances and $Q$-functions (as compared to 8 in the proposed method).

The approximate BER expression can be obtained as in [3], by multiplying each of the terms on the right hand side (RHS) of (12) by $\frac{h_{i, j}}{\log _{2} M}$, where $h_{i, j}$ is the Hamming distance between the symbols $s_{i}$ and $s_{j}$ involved in (11). Using the bit-to-symbol mapping given in [5], we multiply the RHS of (12) with $\frac{1}{6}, \frac{2}{6}, \frac{1}{6}, \frac{1}{6}, \frac{2}{6}, \frac{1}{6}, \frac{2}{6}, \frac{1}{6}$ respectively.

Computer Simulations: Computer simulations were performed to verify the accuracy of the proposed method. Fig. 2 shows the SER and BER plot of the derived expressions, wherein a close agreement with simulation results is obtained. The proposed approach has also been applied to 16- and 32-APSK, with simulation results showing good agreement with the resulting simplified formulas.

Conclusion: A simplified method for evaluating the error performance of APSK constellations in AWGN has been presented based on a close approximation of the Voronoi diagram. The proposed approach has an increasingly lower computational complexity (as compared to the stateof-the-art) as the constellation order increases, whilst achieving close agreement with simulation results.

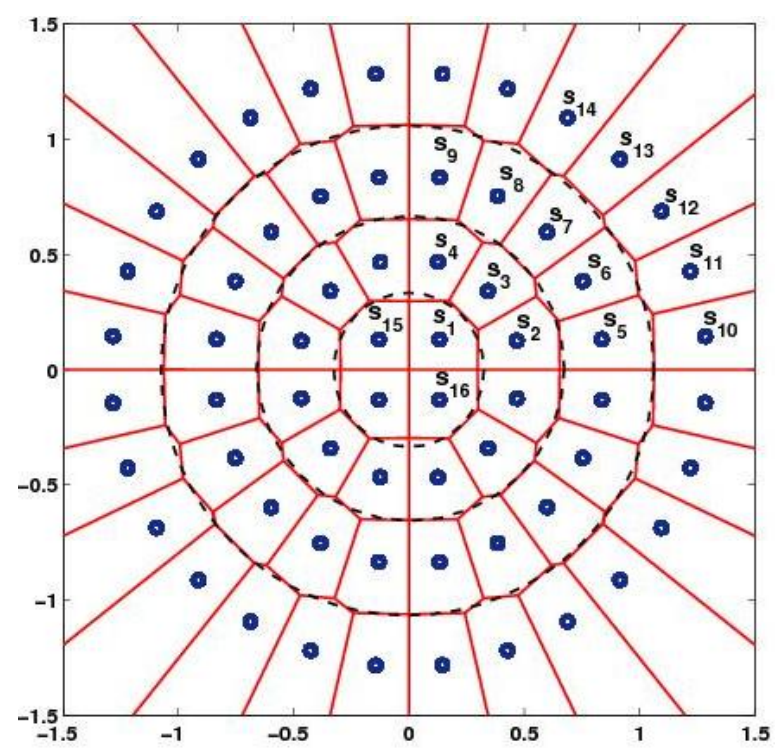

Fig. 1 A Voronoi diagram showing decision boundaries for $M L$ detection in 64-APSK $\left(\gamma_{1}=2.62, \gamma_{2}=4.58\right.$ and $\gamma_{3}=7.00$ [5])

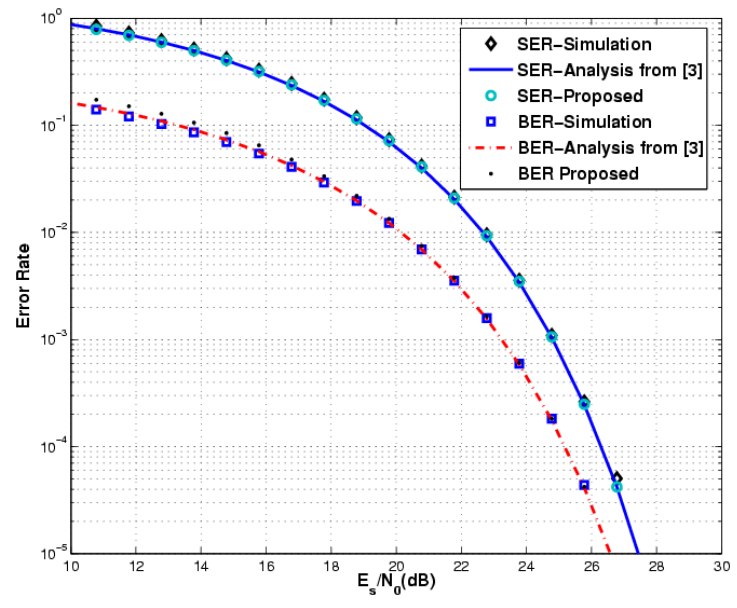

Fig. 2 SER and BER of 64-APSK

\section{References}

1 ETSI EN 302 307: 'Digital Video Broadcasting (DVB); second generation framing structure, channel coding and modulation systems for broadcasting interactive services, news gathering and other broadband satellite applications (DVB-S2)', August 2009.

2 Ziemer, R., and Peterson, R.: 'Introduction to Digital Communications', (2nd Ed), Prentice-Hall, 2001.

3 Sung, W., Kang, S., Kim, P., Chang, D., and Shin, D.: 'Performance analysis of APSK modulation for DVB-S2 transmission over nonlinear channels', Intl. Journal of Satell. Commun. and Net., vol. 27, pp. 295311, May 2009.

4 Miao, G. J.: 'Signal Processing in Digital Communications', Artech House, 2007.

5 Flexible Serially Concatenated Convolutional Turbo Codes with Near- Shannon Bound Performance for Telemetry Applications, CCSDS Orange Book, CCSDS 131.2-O-1, Issue 1, Sept. 2007.

6 Liolis, K. P., and Alagha, N. S.: 'On 64-APSK constellation design optimization', in Signal Processing for Space Communications 10th International Workshop, pp. 1-7, 2008. 